\title{
Erratum
}

\section{Alleviating psychosocial issues for individuals with communication impairments and their families following stroke: A case series of interdisciplinary assessment and intervention}

Ciara Jones, Kiadhnait O’Keeffe, Craig Kingston and Aine Carroll

[NeuroRehabilitation 32 (2013) 351-358]

When this article was originally published, the name of the second author was incorrectly spelled. The correct spelling is Fiadhnait O'Keeffe. 